\title{
Computational study of methyl group dynamics in the hydroquinone clathrate of acetonitrile
}

\author{
Alexandra Nemkevich, Ben Corry and Mark A. Spackman* \\ Received 23rd September 2011, Accepted 1st December 2011 \\ DOI: $10.1039 / \mathrm{c} 2 \mathrm{cp} 23384 \mathrm{c}$
}

We report molecular dynamics simulations of the acetonitrile clathrate of hydroquinone, with a focus on the dynamics of acetonitrile methyl groups. There are three inequivalent acetonitrile molecules in the unit cell, one with its dipole parallel to the $c$-axis, and the other two antiparallel. Although these three guest molecules have previously been found to exhibit two slightly different frequencies of rotation over a wide range of temperatures, the frequencies could not be assigned to specific methyl groups. Perhaps counterintuitively, our simulations suggest that the molecule with the lower frequency is one of the two molecules oriented the same way, the different dynamical behaviour being due to subtle differences in the environments of the molecules.

Hydroquinone (HQ) is well known to form crystalline host-guest systems with small molecules such as $\mathrm{SO}_{2}, \mathrm{H}_{2} \mathrm{~S}$, $\mathrm{HBr}, \mathrm{CO}, \mathrm{CH}_{4}, \mathrm{CH}_{3} \mathrm{OH}$, acetonitrile and methyl isocyanide. ${ }^{1}$ Acetonitrile appears to be one of the largest guests the hydroquinone apohost can accommodate, and the HQ:acetonitrile clathrate is the only one known to crystallize in the $P 3$ space group, ${ }^{2}$ and not in the higher symmetry $R 3$ and $R \overline{3}$ space groups found for other hydroquinone clathrates and for the apohost $\beta$-hydroquinone. ${ }^{3}$ Due to this lower symmetry, the acetonitrile guest molecules occupy three inequivalent cavities in the unit cell, with dipoles oriented $1: 2$ in opposite directions along the crystallographic $c$ axis. ${ }^{2}$

A combined single crystal deuteron NMR and inelastic neutron scattering study of dynamics of acetonitrile methyl groups in HQ:acetonitrile has found two frequencies of rotation for the three inequivalent acetonitrile molecules: at a range of temperatures from below $40 \mathrm{~K}$ to room temperature two acetonitrile molecules rotate slightly faster than the third. The two frequencies could not be assigned to specific methyl groups in the unit cell. ${ }^{4}$ Here we report molecular dynamics simulations of HQ:acetonitrile focusing on the dynamics of acetonitrile methyl groups and providing an answer to the questions of which molecule rotates slower, and why.

The crystal structure of HQ:acetonitrile at $15 \mathrm{~K}$, recently determined by Clausen, ${ }^{5}$ forms the starting point for the MD simulations. The unit cell was replicated in three dimensions to form $2 \times 2 \times 5$ unit cells, or a $31.569 \times 31.569 \times 30.868 \AA$

School of Biomedical, Biomolecular and Chemical Sciences,

University of Western Australia, Perth, Australia.

E-mail:mark.spackman@uwa.edu.au supercell with 60 acetonitrile molecules in total. The system was minimized and equilibrated for $5 \mathrm{~ns}$ at each of $100 \mathrm{~K}$ and $300 \mathrm{~K}$ in the NPT ensemble using the General Amber Force Field (GAFF). ${ }^{6}$ Unfortunately, as anisotropic displacement parameters (ADPs) for atoms in HQ:acetonitrile are only available at $15 \mathrm{~K}$, we are unable to compare the thermal motion from our simulations with that observed experimentally at these temperatures, a benchmark that we have found to be extremely useful in our earlier work. ${ }^{7}$ Average structures of the supercell at $100 \mathrm{~K}$ and at $300 \mathrm{~K}$ were also obtained from the simulations and used to find average volumes of Hirshfeld surfaces for acetonitriles. Hirshfeld surface analysis has recently emerged as an effective tool for identifying and quantifying intermolecular interactions in crystals. ${ }^{8}$ In addition to volumes defined by these surfaces for each acetonitrile molecule, we map the normalised contact distance, $d_{\text {norm }}$, based on distances relative to van der Waals radii between surface points and the nearest nuclei inside and outside the surface. ${ }^{9}$

In the text we refer to acetonitrile molecules $\mathrm{A}, \mathrm{B}$ and $\mathrm{C}$ as those with nitrogen $x$ and $y$ fractional coordinates $(1 / 3,2 / 3)$, $(2 / 3,1 / 3)$, and $(0,0)$, respectively (Fig. 1). Molecule $A$ is oriented in one direction along $c$, while molecules $\mathrm{B}$ and $\mathrm{C}$ are oriented in the opposite direction.

In our simulations at both $100 \mathrm{~K}$ and $300 \mathrm{~K}$ the acetonitrile methyl groups perform $\sim 120^{\circ}$ jumps about the methyl threefold axis. This should correctly reflect the real dynamics at these temperatures, as it was established experimentally that proton tunnelling ceases at $40 \mathrm{~K}$ and the mechanism at temperatures above $40 \mathrm{~K}$ involves classical stochastic jumps between the three equilibrium orientations of the methyl groups, with no contribution from proton tunnelling. ${ }^{4}$ From the MD trajectories, the reorientation frequencies for methyl groups were extracted by counting the number of $\sim 120^{\circ}$ jumps of the methyl groups in each of the 60 molecules in the supercell. The frequencies were then averaged separately for the three inequivalent acetonitrile molecules. Contrary to the obvious inclination to ascribe different frequencies to the molecules oriented in opposite ways, our frequency calculations (Table 1) suggest that the molecule with the lower frequency of reorientation (i.e. with the higher barrier to rotation) is molecule $\mathrm{C}$, which is one of the two molecules oriented the same way. From simulations at both $100 \mathrm{~K}$ and $300 \mathrm{~K}$, molecules $\mathrm{A}$ and $\mathrm{B}$ are found to have very similar methyl rotation frequencies, while those for molecule $\mathrm{C}$ are lower by $22 \%$ at $100 \mathrm{~K}$ and $10 \%$ at $300 \mathrm{~K}$. 

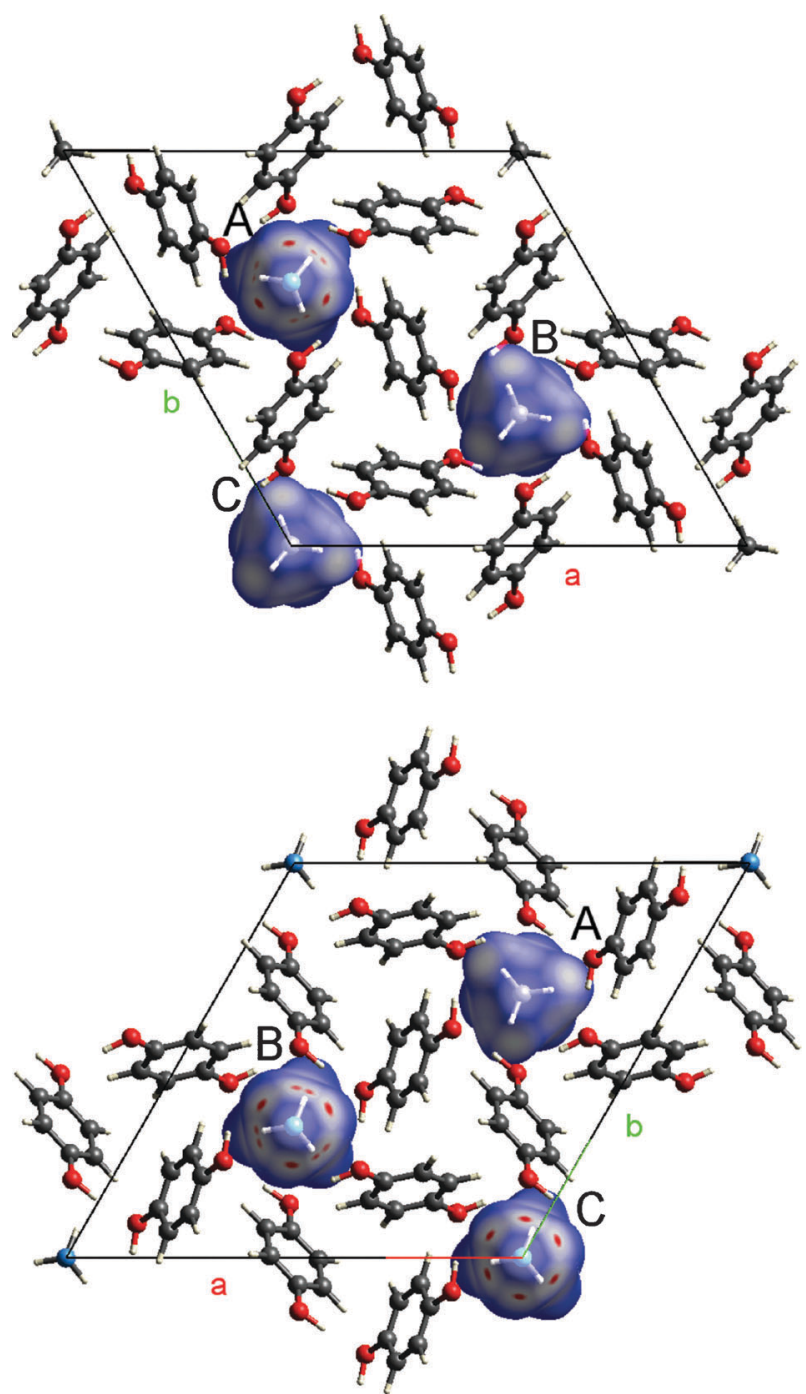

Fig. 1 Visualisation of the HQ:acetonitrile crystal structure at $15 \mathrm{~K}^{5}$ Hirshfeld surfaces for the three acetonitrile molecules are shown with $d_{\text {norm }}$ plotted on the surfaces. Red indicates areas of close contact. The $c$-axis is pointing towards the viewer (top) and away (bottom). Differences in the environments between molecules $\mathrm{A}$ and $\mathrm{B}$ and molecule $\mathrm{C}$ can be seen.

Table 1 Frequencies of methyl reorientation for three inequivalent acetonitrile molecules in hydroquinone clathrate

\begin{tabular}{|c|c|c|}
\hline & $100 \mathrm{~K}\left(\mathrm{~s}^{-1}\right)$ & $300 \mathrm{~K}\left(\mathrm{~s}^{-1}\right)$ \\
\hline $\begin{array}{l}\text { Molecule A } \\
\text { Molecule B } \\
\text { Molecule C } \\
\text { Average } \\
\text { Experiment }\end{array}$ & $\begin{array}{l}(3.1 \pm 0.2) \times 10^{9} \\
(3.3 \pm 0.2) \times 10^{9} \\
(2.5 \pm 0.2) \times 10^{9} \\
3.0 \times 10^{9} \\
\sim 1.3 \times 10^{9}\end{array}$ & $\begin{array}{l}(90.5 \pm 1.1) \times 10^{9} \\
(89.9 \pm 0.8) \times 10^{9} \\
(80.5 \pm 0.9) \times 10^{9} \\
8.7 \times 10^{10} \\
\sim 3.2 \times 10^{11}\end{array}$ \\
\hline \multicolumn{3}{|c|}{${ }^{a}$ Detken et $a l .{ }^{4}$ (see the text). } \\
\hline
\end{tabular}

For comparison with results from our simulations, Table 1 gives experimental estimates at these two temperatures, obtained as the inverse of correlation times of incoherent reorientations from Fig. 7 of Detken et al. ${ }^{4}$ In that study the spin-lattice relaxation times for the inequivalent methyl groups were found to be different by no more than a factor of 3 , which made it difficult to determine the two frequencies independently, so a single correlation time was derived, but the authors recognized that this is not strictly correct. Given the limitations of the force field used in the MD simulations, and the fact that the experimental results were obtained for $\mathrm{CD}_{3} \mathrm{CN}$ rather than $\mathrm{CH}_{3} \mathrm{CN}$, the agreement with experiment is remarkably good.

Based on the $15 \mathrm{~K}$ crystal structure of HQ:acetonitrile, ${ }^{5}$ Hirshfeld surfaces mapped with $d_{\text {norm }}$ for the three acetonitrile molecules (Fig. 1) clearly identify two different environments for the three acetonitrile molecules: molecules A and B exhibit near identical features (i.e. red spots) due to close contacts around the $\mathrm{N}$ atom, while the surface for molecule $\mathrm{C}$ is distinctly different. It is known that the position of molecule A (i.e. the one oriented opposite to the other two) in the cavity is different to the position of molecules $\mathrm{B}$ and $\mathrm{C}^{2}$ but other geometric factors make the environment of molecule $\mathrm{C}$ different from those of molecules A and B. Hirshfeld surface analysis of the three acetonitrile molecules confirms that the majority of close contacts made by the methyl groups are with the hydrogens of the six-membered hydrogen bonded rings separating adjacent cavities, and formed by hydroxyls of hydroquinone molecules. As observed previously by Detken et al., ${ }^{4}$ these interactions are expected to be important in determining the rotational potential of the methyl groups. At $15 \mathrm{~K}$ the geometry of these rings is subtly different between channels occupied by molecules A and B and channels occupied by molecule $\mathrm{C}$. For example, in channels of molecules $\mathrm{A}$ and $\mathrm{B}$ alternate hydrogen atoms protrude further from the mean plane of the six oxygens, and into the cavity towards the acetonitrile nitrogen atom, and hence further from the methyl hydrogens of an adjoining acetonitrile, while the other three hydroxyl hydrogens of the ring lie almost in the mean plane. Thus the $\mathrm{O}-\mathrm{H}-\mathrm{O}$ angles in the ring alternate between $164.4^{\circ}$ and $174.7^{\circ}$, and the $\mathrm{O}-\mathrm{O}$ distances alternate between $2.71 \AA$ and $2.74 \AA$. In contrast, in channels with molecule $\mathrm{C}$ hydroxyl hydrogens protrude alternately above and below the mean oxygen plane, and at similar distances from the plane, with all $\mathrm{O}-\mathrm{H}-\mathrm{O}$ angles equal to $168.9^{\circ}$ and all $\mathrm{O}-\mathrm{O}$ distances $2.73 \AA$. On the $d_{\text {norm }}$ surface (Fig. 1) the patterns of red spots designating areas of close contacts are the same for molecules $\mathrm{A}$ and $\mathrm{B}$ (and reflect the alternating arrangement of hydroxyl hydrogens), but quite different for molecule C. While these features focus on the environments of nitrogen atoms, the underlying cause (i.e. the differences in the ring geometries) necessarily affects the methyl groups on the other side of the hydrogen bonded ring. In addition to these slight differences in geometries of the six-membered hydrogen bonded rings, small differences are also evident in the distances of methyl hydrogens to aromatic hydrogens, and together they result in a slightly smaller volume available to molecule $\mathrm{C}$ compared to molecules $\mathrm{A}$ and $\mathrm{B}$, as can be seen in the volumes of Hirshfeld surfaces for the three molecules (Table 2).

As the temperature rises, the unit cell expands and increasing thermal motion of atoms results in smaller relative differences in the methyl rotational frequencies between molecules $\mathrm{A}$ and $\mathrm{B}$, and that for $\mathrm{C}$, at $300 \mathrm{~K}$ compared to $100 \mathrm{~K}$ as already noted. The environments of molecules $\mathrm{A}$ and $\mathrm{B}$ change in slightly different ways, as reflected by the volumes of their Hirshfeld surfaces; in our simulations at both temperatures the volume for molecule $\mathrm{C}$ is marginally smaller (Table 2). It is impossible to pinpoint a single geometric factor that would 
Table 2 Volumes of Hirshfeld surfaces $\left(V_{\mathrm{H}}\right)$ in $\AA^{3}$ of three inequivalent acetonitrile molecules based on the crystal structure at $15 \mathrm{~K}^{5}$ and average crystal structures from simulations at $100 \mathrm{~K}$ and at $300 \mathrm{~K}$

\begin{tabular}{lllll}
\hline & \multirow{2}{nnnn}{} & Crystal structure & & \multicolumn{2}{l}{ Simulation } & \\
\cline { 2 - 2 } \cline { 5 - 5 } & $15 \mathrm{~K}$ & & $100 \mathrm{~K}$ & $300 \mathrm{~K}$ \\
\hline Molecule A & 59.3 & 61.6 & 64.0 \\
Molecule B & 59.3 & 61.8 & 64.3 \\
Molecule C & 59.0 & 61.4 & 63.7 \\
\hline
\end{tabular}

account for the slightly smaller volume of Hirshfeld surfaces for molecule C. Rather we expect it to be a cooperative effect of tiny differences in many geometric parameters, resulting from the need for the host to accommodate a network of molecules with large dipole-dipole interaction energies. Changes in the crystal structure of HQ:acetonitrile as a function of temperature may identify key geometric factors. Unfortunately we only have accurate and precise structural information at $15 \mathrm{~K}$, as the earlier room temperature structure ${ }^{2}$ did not locate the methyl hydrogen positions. Whatever these geometric factors may be, on average, hydrogen atoms of molecule $\mathrm{C}$ experience a stronger repulsive force, indicative of closer contacts, which in turn results in a higher barrier and lower frequency of rotation.

In summary, solid state NMR has previously identified two slightly different frequencies for methyl group rotation in the three inequivalent acetonitrile molecules in the HQ:acetonitrile clathrate, but was unable to pinpoint which molecule is different. From MD simulations and careful analysis of the average structures, we conclude that the methyl group with the lesser rotation frequency is one of the two molecules oriented in the same direction along the $c$-axis. The underlying reason for this difference in dynamic behaviour of the acetonitrile molecules is the slightly different environment experienced by each in the crystal. Earlier force-field calculations ${ }^{4}$ did not agree qualitatively with the experimental findings, in that they predicted two molecules to have a higher rotational barrier than the third, while in the experiment it is the other way around. In contrast, our results agree qualitatively with this experimental observation, as well as quantitatively with experimental frequencies of rotation. Along with our earlier work $^{10}$ this study illustrates how MD simulations can be used to study fine structural and dynamic details of quite complicated molecular crystals, nicely complementing the experiments while characterising dynamic properties that are often difficult to analyse experimentally.

\section{Acknowledgements}

The authors thank the Australian Research Council for financial support. AN acknowledges the Australian Government for an International Postgraduate Research Scholarship and the University of Western Australia for a University Postgraduate Award. Computer time was allocated under the merit allocation scheme of the National Computational Infrastructure (Australia).

\section{References}

1 T. C. W. Mak and C. K. Lam, in Encyclopedia of Supramolecular Chemistry, ed. J. L. Atwood and J. W. Steed, Marcel Dekker, New York, 2004, pp. 679-686.

2 T. L. Chan and T. C. W. Mak, J. Chem. Soc., Perkin Trans. 2, 1983, 777-781.

3 H. F. Clausen, Y.-S. Chen, D. Jayatilaka, J. Overgaard, G. A. Koutsantonis, M. A. Spackman and B. B. Iversen, J. Phys. Chem. A, 2011, 115, 12962-12972.

4 A. Detken, P. Schiebel, M. Johnson, H. Zimmermann and U. Haeberlen, Chem. Phys., 1998, 238, 301-314.

5 H.F. Clausen, 2011, private communication.

6 J. M. Wang, R. M. Wolf, J. W. Caldwell, P. A. Kollman and D. A. Case, J. Comput. Chem., 2004, 25, 1157-1174.

7 A. Nemkevich, H. B. Buergi, M. A. Spackman and B. Corry, Phys. Chem. Chem. Phys., 2010, 12, 14916-14929.

8 M. A. Spackman and D. Jayatilaka, CrystEngComm, 2009, 11, 19-32.

9 J. J. McKinnon, D. Jayatilaka and M. A. Spackman, Chem. Commun., 2007, 3814-3816.

10 A. Nemkevich, M. A. Spackman and B. Corry, J. Am. Chem. Soc., 2011, 133, 18880-18888. 